\title{
Wind Direction Readouts
}

\author{
Ronald L. Ives \\ Metronics Associates, Inc., Palo Allo, Calif.
}

18 October 1965 and 15 February 1966

\section{Introduction}

For considerably more than a generation, the combination of a contacting wind vane, a contacting anemometer, and a multi-lamp wind speed and direction indicator ${ }^{1}$ has been the standard wind measuring and indicating system at most weather stationscivil, military and industrial. Because the instruments are rugged, and designed on sound principles, they will probably continue in wide use for at least another generation.

Within the last decade or so, however, as the number of dials and indicators to be watched by the meteorologist has increased, the rugged and bulky nine-lamp wind indicator has become a problem, as it takes up a considerable volume, and is incompatible with the other dials and indicators currently in use.

At least as long ago as 1942, operational equivalents of the ML-117 wind indicator, having considerably less bulk, were constructed by use of electronic indicator lamps. Recently, with the development of "in-line" readouts, a highly legible wind clirection display can be constructed to occupy a panel space of only $1 \frac{1}{2}-$ by 3 inches (or less with less readability). Appearance of such a display is shown in Fig. 1. A standard display consists of twelve lamps, twelve messages, a lens system and a screen. When the lamp associated with a given message is lighted, that message shows on the

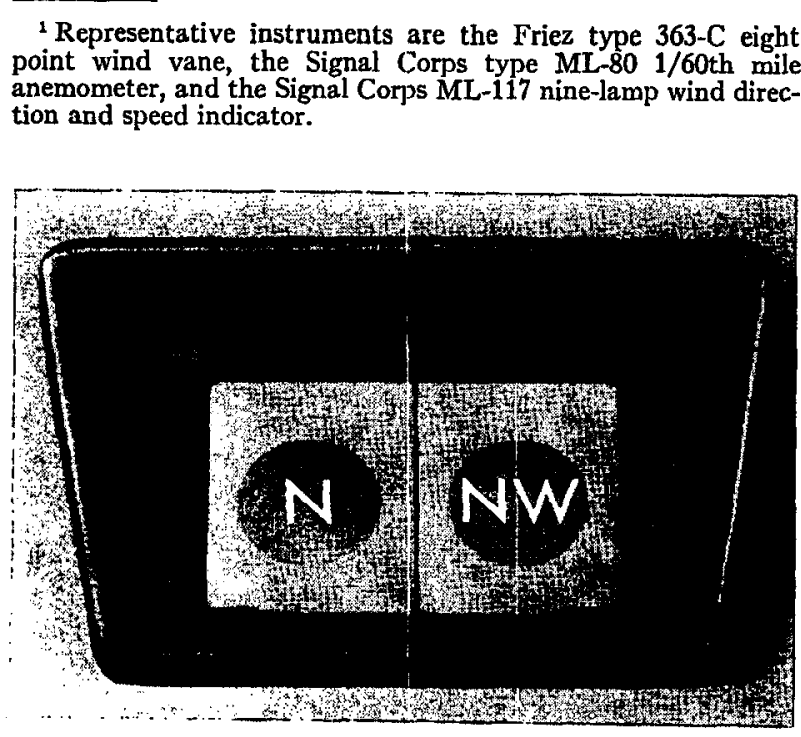

FIG. 1. Wind direction display used in in-line readouts. screen. A wide variety of lamps can be used in these readouts ${ }^{2}$. Functionally, the in-line readout is a battery of 12 miniature projection lanterns.

\section{Two readout system}

Customarily, wind direction is transmitted by an eight-point contacting wind vane which is indicated to sixteen points by overlapping of the contacting sectors. Connection of a conventional wind vane to two readouts, to give sixteen-point wind direction indication is shown in Fig. 2. Circuitry is entirely standard and conventional. Lamps here are operated from a high voltage supply, in series with a dropping resistor, to extend their life by eliminating current surges when they are switched. With this procedure, catastrophic lamp failures are virtually eliminated, and replacements are normally made every six months. Position of wind vane contactor shown in this figure gives the NNW indication shown in Fig. 1.

\section{Single readout system}

Where only eight point direction indication is needed, and overlaps are undesirable, a single readout can be used with a relatively simple transistorized anti-overlap circuit. With this arrangement, when two vane contact sectors are energized, the lamp connected to the one furthest clockwise lights, and the circuit of the trailing lamp is opened by the transistor. The circuit is shown in Fig. 3 where both the Northwest and North contact sectors are energized by the vane contactor. Positive potential is applied to the upper end of the North and Northwest lamps, and to the bases of the transistors associated with the Northwest and West lamps.

The transistors used here are of the PNP type, which conduct strongly when the base is negative with respect to the emitter, and are virtually an open circuit when the base is positive relative to the emitter. In the present situation, the Northwest transistor, being biased positively from the North vane sector, is nonconducting, so that the Northwest lamp cannot light. The base of the North transistor, in contrast, is tied to the system negative, and no positive bias is applied. In consequence, it conducts, permitting the North lamp to light.

${ }^{2}$ Readouts used and referenced are IEE Series 10, made by Industrial Electronic Engineers, Inc., 7720 Lemona Ave., Van
Nuys, Calif. 


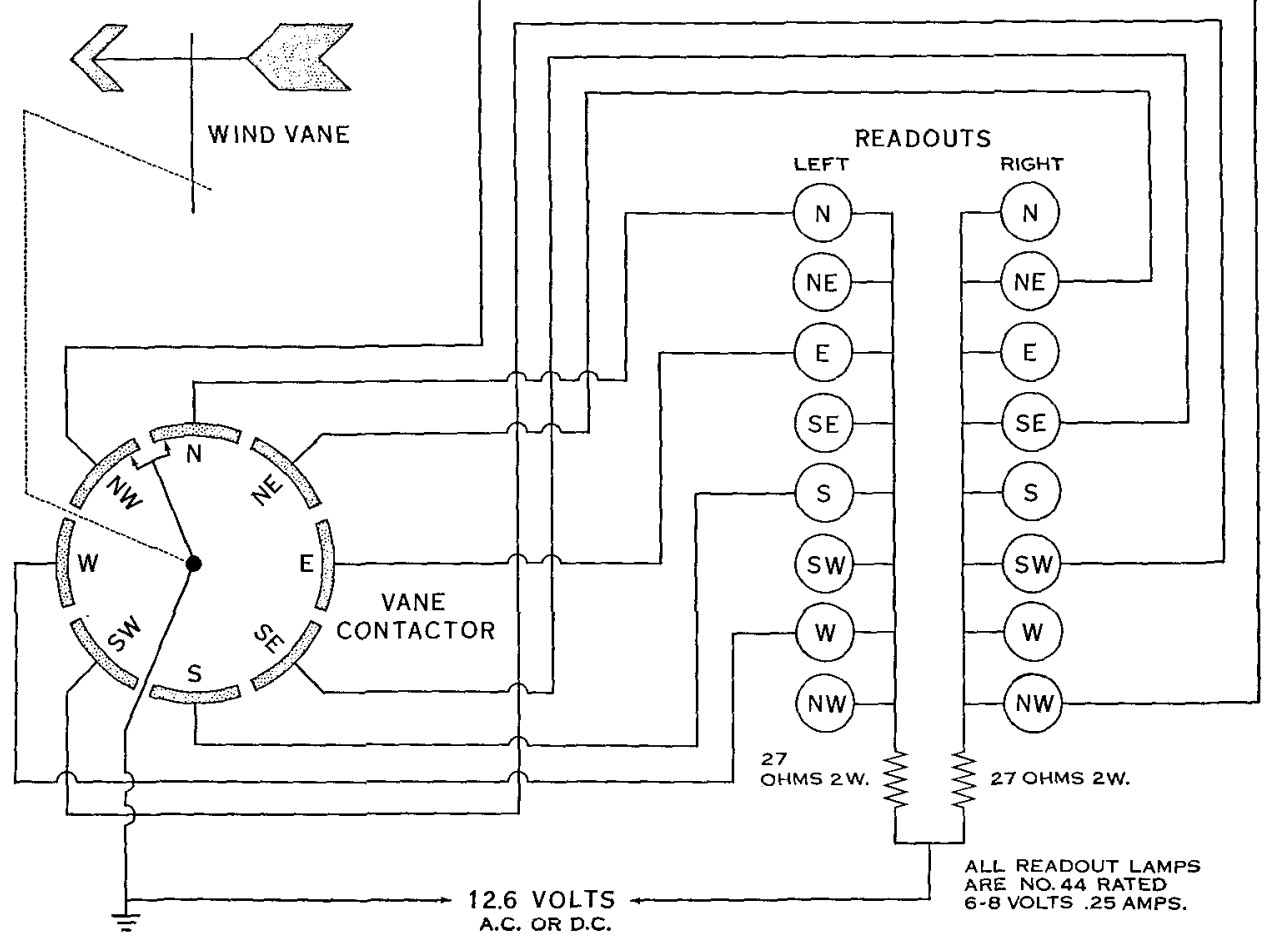

FIG. 2. Circuit of two-readout system to give sixteen-point wind direction indication.

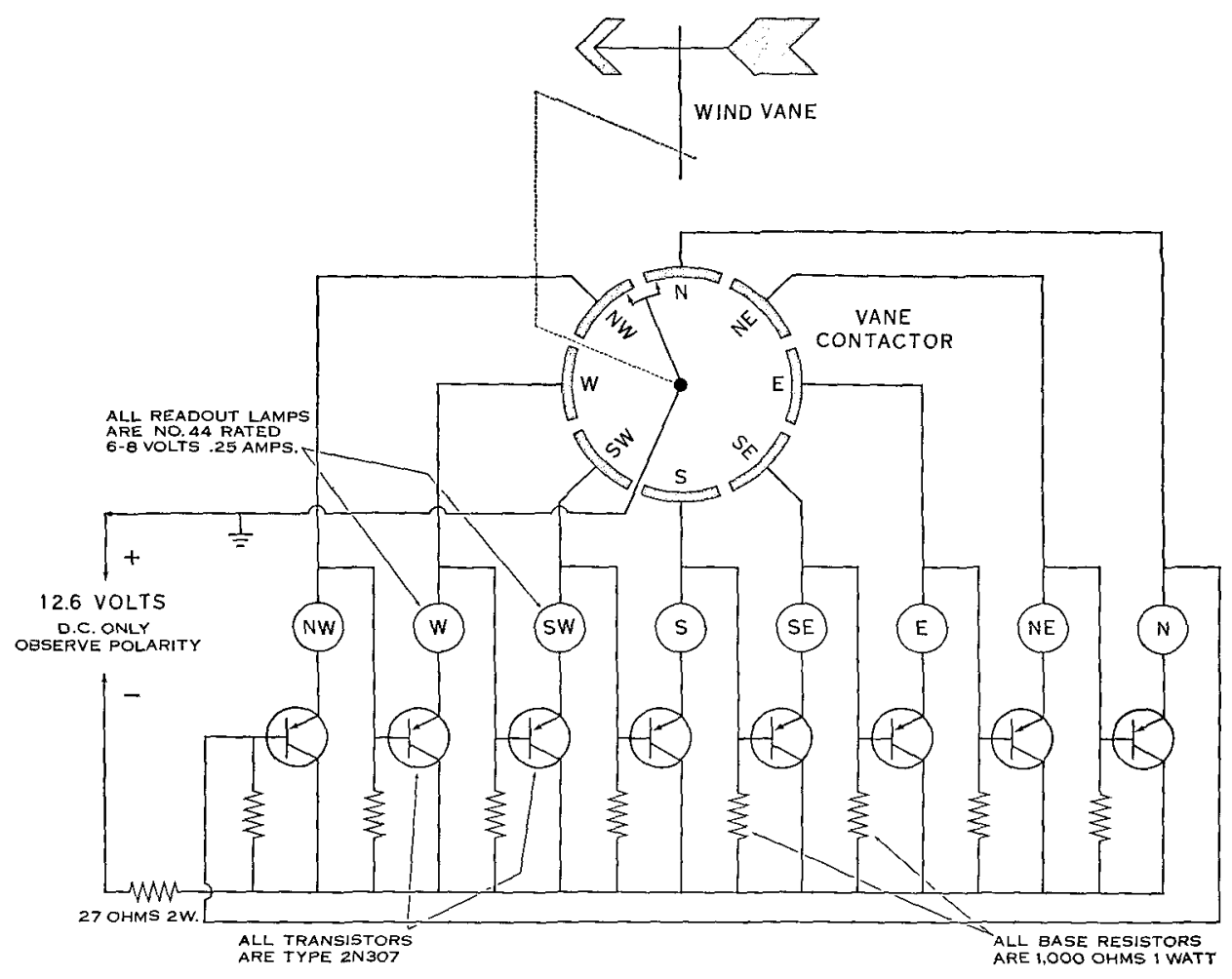

FIG. 3. Circuit of single-readout eight-point indicator. 


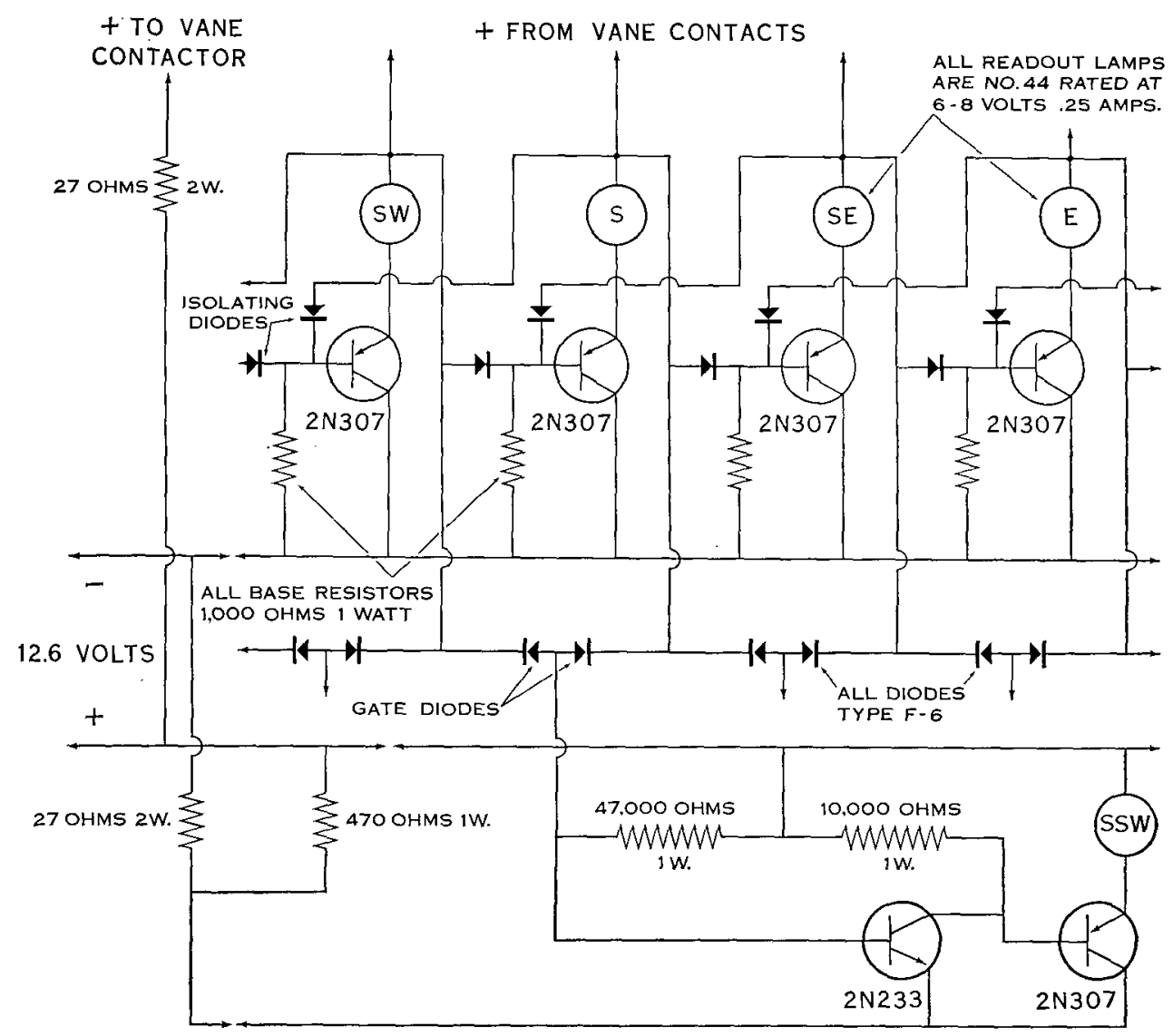

FIG. 4. Partial circuit of sixteen-point single-readout system.

A slight directional inaccuracy is introduced into the system by the "one way" elimination of overlaps, and is corrected for by a minor reorientation of the vane assembly. By a mirror reversal of connections, the most counterclockwise lamp circuit can be favored, with system operation otherwise unchanged. Several rather involved circuits have been developed to permit eight point indication from an eight contact vane without correction of orientation.

\section{Sixteen-point single-readout system}

Indication of wind direction to sixteen points, from an eight contact vane, without overlap problems, requires the use of a sixteen lamp readout and some simple logic circuitry. A partial circuit of the sixteen-point single-readout system comprises Fig. 4. Omitted sections are identical to those shown except for direction designations. Here, the readout lamps are divided into two sequences. When any single lamp in the main sequence (North, Northeast, East, Southeast, etc.) is energized from the vane contacts, that lamp lights, the base of its associated transistor being tied to system negative, and no positive bias is applied from any source.
At the same time, the bases of the transistors associated with the two immediately adjacent lamps are biased positively, so that they cannot conduct, while the cathodes of the two gate diodes associated with the energized lamps are also biased positively ("blocked"), so that they cannot conduct under the conditions prevailing.

The isolating diodes shown here prevent unwanted circuit interconnections ("sneak circuits"). Were they absent, energization of any one lamp circuit would result in illumination of not only that lamp, but also of three others, alternately arranged. For example, with no isolating diodes, energization of the South lamp would result in the illumination of the South, West, North and East lamps.

When two adjacent vane sectors are energized, neither associated lamp in the main sequence lights, for each biases the other off. Also, the gate diodes between the two circuits are blocked, so that they cannot conduct under existing circuit conditions.

Lamps in the intermediate sequence (North-northeast, East-northeast, East-southeast, South-southeast, etc.) are indirectly controlled by their respective pairs of gate diodes. Each lamp in the intermediate sequence is connected across the line in series with a PNP tran- 
sistor. The base of this transistor is tied to system positive through a resistor, so that the transistor normally does not conduct, and the lamp, in consequence, remains unlighted.

From the base of the lamp control transistor (2N307) to local system negative, in each case, an NPN transistor is connected. This conducts when the base is biased positively relative to the emitter, and is nonconducting when the base is biased negatively. Positive bias for this transistor (2N233) is available from system positive through a high resistance, but this bias is normally bled away through the gate diodes, so that neither transistor is conducting. Only when both diodes connected to the base of the 2 N233 are blocked can positive bias get to its base, permitting it to conduct. When this occurs, and at no other time, the base of the $2 \mathrm{~N} 307$ is connected to local system negative, so that it, in turn, can conduct, completing the circuit through the indicator lamp, which then lights.

In the circuit shown, requisite conditions for lighting the South-southwest lamp occur only when the Southwest and West sectors of the vane contacts are energized by the contactor.

Several workable alternatives to this circuit are possible, this one being preferred at the present state of the art because it works consistently using standard and easily available components. This circuit replaces the costly, noisy and trouble-prone battery of relays used a quarter of a century ago to perform the same function.

\section{Alternative operations}

Although specifically designed for operating readout lamps from a contacting wind vane, these same circuits can be used with equal success for controlling and operating other electrical loads, such as counters, relays and chronograph pens.

\section{Performance and service life}

At the present state of the art, the most fallible components in the readout systems outlined are the indicator lamps, which have a nominal life exceeding 1000 hours of continuous service. Lamp life is radically shortened by switching, but most of its ill effects can be reduced by operating the lamps from a high voltage supply in series with a suitable voltage-dropping resistor. This, by reducing turn-on current surges, extends lamp life and virtually eliminates catastrophic failures (burn-outs). Replacements can be made at scheduled times, such as every six months, as light output of the lamps declines due to bulb blackening.

Transistors and diodes have a problematical, but very long, service life, considerably exceeding five years of continuous service with the constants and loads here designated. Other transistors, having about the same characteristics as those specified, will work quite well in these circuits. Manufacturer's heat sink specifications should be followed carefully.

\section{Conclusions}

Due to advances in the electronic art, the bulky and awkward, but highly dependable ML-117 wind indicator can be replaced by a compact and convenient readout, having at least the same dependability.

Numerous special functions, formerly requiring large banks of relays, which are bulky, noisy and demanding of maintenance, can now be performed by compact, silent and substantially trouble-free diode and transistor circuits.

Improvements in lamp supply circuitry change bulb replacements from a recurrent emergency to a schedulable routine maintenance operation.

\title{
An Economical Multiplexer for Use on Radiosondes
}

\author{
R. W. Kobussen and J. A. Wetnman \\ University of Wisconsin, Madison
}

23 December 1965 and 4 April 1966

\section{Introduction}

The need to place more than two sensors aboard a 1680-megacycle radiosonde requires either that more contact points be incorporated in the baroswitch, or that a separate switching device be added to the sonde. Adding more contact points to the baroswitch is not feasible because of dimensional limitations. A switch, consisting of copper strips printed on a circuit board and a wiper arm which contacts one strip at a time as the wiper rotates, is currently used when 